\title{
De Hanoi à Saigon par le chemin des écoliers
}

Le journal de vacances de Claudie Beaucarnot (1943)

\section{Ellen Furlough}

\section{(2) OpenEdition \\ Journals}

Édition électronique

URL : https://journals.openedition.org/clio/8702

DOI : $10.4000 /$ clio.8702

ISSN : 1777-5299

Éditeur

Belin

Édition imprimée

Date de publication : 15 décembre 2008

Pagination : 204-212

ISSN : 1252-7017

Référence électronique

Ellen Furlough, «De Hanoi à Saigon par le chemin des écoliers », Clio. Histoire, femmes et sociétés [En ligne], 28 | 2008, mis en ligne le 15 décembre 2011, consulté le 22 avril 2022. URL : http:// journals.openedition.org/clio/8702; DOI : https://doi.org/10.4000/clio.8702 


\title{
De Hanoi à Saigon par le chemin des écoliers Le journal de vacances de Claudie Beaucarnot (1943)
}

\author{
Ellen FURLOUGH
}

Préface [Mars 1990] Pour être agréable à une amie du Lycée Albert Sarraut à Hanoi qui voulait rassembler des documents afin de constituer un témoignage sur la vie quotidienne des Français en Indochine jusqu'en 1945, j'ai fouillé dans mes papiers conservés en vrac dans un carton. J'y ai retrouvé ces notes écrites sur trois petits carnets, au jour le jour, dans la voiture qui nous emmenait pour ces vacances 1943 . Je les avais oubliés depuis trente ans! [...] Je ne me doutais pas que deux ans après cette toute simple relation de voyage, notre monde à nous, les Français d'Indochine, s'écroulerait le 9 Mars 1945. [...] Il a fallu que nous nous trouvions à ce moment précis de l'histoire de l'Indochine qui vit notre éviction de ce pays ! Pourtant ce pays était le mien. Mon père y était venu tout jeune, peu après la guerre de 1914, en 1920. Parti comme Directeur Général des Tuileries de l'Indochine, il l'est resté durant toute sa vie indochinoise. Sillonnant l'Indochine du Nord au Sud sans relâche, il découvrit de nombreux minerais qui permirent des fabrications nouvelles pour la mise en valeur des ressources de ce pays. Connaissant bien l'Indochine, il l'aimait et en estimait ses habitants. Il prit pour épouse, maman, née d'un père breton, arrivé à Hanoi vers 1890 comme professeur de mathématiques, et d'une mère vietnamienne d'une bonne famille de Ha Dong. Tous les éléments étaient donc réunis pour que nous soyons de vrais Indochinois et que nous restions toute notre vie dans ce pays qui était le nôtre. Les événements nous en chassèrent. Ce fut un déchirement total.

Lundi 28 juin [1943] : Date mémorable : nous ne sommes jamais restées si tard à Hanoi. D'habitude, dès le 15 juin, après la distribution des prix de notre cher vieux Lycée Albert Sarraut, nous partons (maman et ses filles) pour le Tam Dao à la fraîcheur [...] Par un heureux hasard, cette année [...] papa doit se rendre en Cochinchine pour inspecter la tuilerie de Long Buu et chercher de nouvelles terres, argiles, et minerais afin de mettre en route de nouvelles fabrications. Nous en profitons donc pour partir en famille et joindre l'utile à l'agréable. Nous voilà donc «Sur la Route Mandarine » que Roland Dorgelès a 
décrite avec tant de grâce ! [...] Nous avons produit notre petit effet à la sortie d'Hanoi. Je sais bien que depuis la guerre, les Hanö̈ens voient circuler dans leur bonne ville les véhicules les plus extraordinaires. Cependant, nous leur en avons mis plein la vue avec notre gazogène [...]. Après Phu Ly, si nous tournions a droite, nous arriverions à Chiné, à une quarantaine de kilomètres, la plantation de café de papa Leconte. Puis à Dong Lang, sa ferme. C'est là que sont les immenses séchoirs à café et tout le matériel propre à traiter le café, et à l'ensacher pour l'expédier un peu partout, surtout vers la France avant la guerre. Dong Lang, au milieu des montagnes calcaires est situé en Moyenne Région et peuplée d'une ethnie : les Muongs [...] Enfin Vinh ! Le premier hôtel où nous nous arrêtons est complet! Un deuxième hésite à nous recevoir. Après bien des palabres, on nous met à l'annexe. C'est propre. Il y a une douche dont nous usons avec volupté $[\ldots]$

Mardi 29 juin Le décor a changé. Ce ne sont plus des rizières, mais devant nous se dresse une chaîne montagneuse. Sur la crête de la montagne court une muraille. À peu près au milieu de la chaîne, on voit une porte. C'est la Porte d'Annam. Papa m'explique : «Tu vois, de ce côté de la muraille, nous sommes encore en pays d'Annam. De l'autre côté c'est le pays Cham.» [...] Pour passer le temps, nous grignotons tout ce qui nous tombe sous la dent. La boîte de biscuits voit disparaitre rapidement tout son contenu. Nous dévorons également les crêpes et le saucisson annamite acheté ce matin dans un petit village où nous nous sommes arrêtés pour nous procurer du charbon. Cinquante pour cent au moins des gens de ce village avaient les yeux chassieux. C'est qu'ils sont très sensibles à la conjonctivite, et si un membre de la famille l'a, tous les autres l'auront aussi. Ils entouraient la voiture et contemplaient d'un air curieux ces ong tä̈, ba tä̈ qui mangeaient du do an An Nam [...] Cinq kilomètres avant Hué, il y a des paillotes tout le long de la route. Voilà enfin la Citadelle à gauche. Et à droite, la Rivière des Parfums. Hué n'est pas astreinte à l'éclairage de guerre. Il y avait longtemps que nous n'avions vu de lampes non voilées. Cela fait plaisir. Nous pénétrons dans la cour de l'hôtel Morin à neuf heures tapantes. Nous trouvons deux chambres avec beaucoup de peine. Après une bonne douche, nous descendons au restaurant $[\ldots]$

Mercredi 30 juin Cet après-midi, nous irons visiter les tombeaux des empereurs d'Annam [...] Je suis la seule de la famille à ne pas les connaitre, n'ayant jamais eu le temps de les visiter malgré de nombreux passages à Hué... Nous revenons à Hué enchantés de notre promenade. Nous dînons et allons nous coucher. Le cinéma Morin est à deux pas de notre chambre. On y joue Le coeur en fête, un film américain que nous avons déjà vu à Hanoi. Avec Nicole, nous commentons : «tiens, elle (l'héroïne) est en train de faire ceci, 
elle est sur le balcon et chante Siboney ». Puis c'est la scène de la forêt. Enfin, c'est la Sérénade de Schubert dans un décor ridicule de music-hall. Nous nous endormons au son de la Marseillaise et de l'hymne annamite qui terminent la séance.

Samedi 3 juillet L'étape Quang Ngai - Nha Trang est vraiment la plus pittoresque. Encore une courbe. On a une vue aérienne de Sông Câu, de la rade, des rizières en gradins (argiles noires plastiques réfractaires alumineuses). Toutes les montagnes sont cultivées. Toutes les parcelles de terre sont compartimentées. On dirait le bocage vendéen. Ce sont des terres basaltiques. Et cela ressemble aussi au Morvan de papa (col de Gioc Gang). Je rêve et pense à mes grands-parents en France, justement dans le Morvan [...] Nous tombons de fatigue en arrivant chez nos amis. Demain, nous irons à la messe de 6 h30 pour ne pas avoir à sortir les robes de la valise. C'était l'anniversaire de maman et en même temps celui de l'ami chez qui nous sommes descendus. Ils se sont bécotés et nous les avons bécotés.

Jeudi 6 juillet Nous voici près de Phan Rang, en plein pays Cham. On voit deux tours chames au bord de la route [...] Aux abords de Phan Rang, il y a de vastes étendues couvertes de rizières [...] À l'entrée de la ville, le stade. Tout le long de la route, nous avons vu des stades, même dans des villages minuscules. II y a un tas de femmes chames en ville. Pendant qu'on recharge le gazo, elles s'approchent de la voiture pour nous voir. Elles ont toutes une grosse charge de bois sur la tête ou bien un gros panier plein de victuailles. Elles portent des tuniques vertes, jaunes, bleues ou noires. Elles sont très sales et se mettent sur la tête un genre de turban constitué d'une vulgaire serviette de toilette. Ce turban sert de coussinet sur lequel elles posent leurs charges (influence hindoue) [...] Nous avalons une soupe chinoise dans la gargote du coin et nous repartons [...] Puis nous montons vers l'Arbre Broyé. Des Moïs travaillent à la route. Ça me fait un drôle d'effet de les voir travailler pour des étrangers, eux si fiers et indépendants. C'est une race que j'aime beaucoup : ils sont très loyaux et francs. [...] Nous arrivons à l'Arbre Broyé, but de notre voyage et où nous devons passer quelques jours dans un chalet que nous avons loué pour les vacances. Il y a des cabanes en bois. [...] Mais l'intérieur est mignon, propre et coquet. Tout est clair : les meubles, les rideaux aux fenêtres, etc.

Mercredi 7 juillet À $9 \mathrm{~h} 00$ nous partons pour Dalat faire quelques emplettes car il nous manque pas mal de choses [...] Nous sommes arrêtés devant une boutique chinoise où des Moïs se ravitaillent. Ils mettent toutes leurs provisions dans de grandes jarres vernissées qu'ils portent sur leur dos à l'aide d'un support. Il y a là des hommes et des femmes. Ils fument tous la pipe. 
Quand elles ne fument pas, les femmes portent leur pipe dans leur chignon [...] Les hommes ont une ceinture d'étoffe autour des reins et qui leur passe entre les jambes. La ceinture est plus large devant, n'étant plus qu'une ficelle qui entre dans les fesses. C'est leur seul vêtement. [...] Ils sont tous très bronzés et très musclés. Quelle belle race !...

Mardi 13 juillet Ce matin, nous sommes descendus en voiture à Dran. [...] Papa m'a demandé pendant que nous montions la côte, pourquoi je n'aimais pas tellement cette région que lui apprécie beaucoup, car ce paysage lui rappelle son pays natal. Moi, je suis tonkinoise : j'aime mon delta, la plage, la campagne et la mer. Surtout la mer. Enfin tout ce qui est plat.

Vendredi 16 juillet J'ai très mal dormi, énervée, surexcitée et ravie à l'idée d'aller à Saigon. C'est une ville que j'aime beaucoup. J'y ai vécu plus d'un an vers 1936-1937 [...] Saigon a toujours eu un grand attrait pour moi. La vie est très différente, plus gaie, plus nonchalante qu'à Hanoi. Cela tient sans doute au climat et au caractère de ses habitants. Ils prennent la vie du bon côté. D'ailleurs la vie est beaucoup plus facile pour eux qu'au Tonkin. Le pays est riche : le riz pousse en grande quantité dans le delta du Mékong et il y a un tas d'autres cultures intéressantes. Alors qu'au Tonkin, les conditions climatiques sévères obligent les habitants à un travail perpétuel avec un rendement moindre. Le delta du fleuve Rouge est triste. Tout est ocre : le ciel, la terre, les rizières, et même les vêtements des gens couleur cu-nau (brown). Mais j'aime mon Tonkin plus que tout. J'y suis née. Et bien qu'il me soit agréable de le quitter de temps en temps, je suis si heureuse d'y revenir! Hanoi est une ville calme, tranquille, un peu guindée. Saigon est une ville légère, gaie, cosmopolite $[\ldots]$

Vendredi 6 août Partons tous en voiture à huit heures pour Bellevue $[\ldots]$ Pendant la sieste, je fais mon courrier, puis nous mangeons un chào gà, soupe annamite très fine : c'est un bouillon de riz avec des filaments de chair de poulet. C'est un potage rustique que l'on ne propose pas dans les restaurants, mais exquis dans sa simplicité. J'adore la cuisine annamite. À mon avis, c'est la meilleure du monde. Moins grasse que la cuisine chinoise. Je n'ose la comparer à la cuisine française, n'en connaissant que les mets préparés par nos beps indochinois qui tiennent eux-mêmes leur art de dames françaises. Bref, je me nourrirais bien entièrement à la vietnamienne si je pouvais, mais papa ne serait pas d'accord. Il n'a jamais pu s'habituer au nuoc-mâm, la sauce nationale qui est une saumure de poisson et dont l'odeur peut effaroucher des narines occidentales. 
Mardi 31 août Nous allons entrer au Tonkin. La voiture a l'air de sentir l'écurie. Il me semble qu'elle roule plus vite. À Ninh Binh, arrêt chez nos amis qui nous offrent un rafraîchissement. Nous avons hâte de repartir. C'est maintenant le bac de Do Len. Plus loin, Phu Ly. Nous approchons du but. Enfin! C'est Hanoi. Et la voiture s'arrête pile devant le 180 Avenue du Grand Bouddha. Nous revoilà chez nous.

Vacances 1943, ou Hanoi-Saigon par le chemin des écoliers Centre des archives d'outre-mer (67 APOM, d. 2, «Fonds Biggi»)

À la demande de son amie Yvonne Fontain-Biggi du Lycée Albert Sarraut de Hanoi, qui est alors à la recherche de mémoires de personnes ayant séjourné en Indochine, Mme Claudie Brugière (née Beaucarnot) envoie en 1990 une transcription dactylographiée de son journal de vacances de 1943, déposée ensuite, comme les autres documents, au Centre des archives d'outre-mer. Ce tapuscrit de 85 pages contient le récit journalier d'un voyage familial de deux mois, consigné à l'origine sur trois petits cahiers d'écoliers ${ }^{1}$. À dix-neuf ans, la jeune Claudie Beaucarnot raconte le voyage de sa maison de Hanoi au bungalow de vacances près de Dalat, voyage entrecoupé de séjours prolongés à Saigon et à Nha Trang au bord de la mer de Chine pour des vacances balnéaires entre amis. L'expérience du voyage renforce chez la jeune fille le sentiment que sa famille appartient aux élites coloniales, provoque des observations sur les différences culturelles et régionales et favorise des réflexions sur sa propre identité, qu'elle qualifie de «Tonkinoise $»^{2}$.

Née en 1924 au Tonkin d'une famille aisée avec domestiques, Claudie Beaucarnot grandit au sein de ce qu'elle caractérise, dans la

1 L'original du journal reste en possession de Mme Beaucarnot. Une version en ligne en deux langues, réalisée par le Professeur David del Testa, est consultable sur le site suivant: http://www.bucknell.edu/Beaucarnot/intro.shtml (copyright 2002). Mes remerciements à David Del Testa de m'avoir autorisée à reproduire des extraits de la version française. La traduction en anglais a été effectuée par Amber Hart et Stephanie Albee, pour être utilisée avec des étudiants américains. Voir Cahier d'images, figure 9.

2 Le récit de voyage présenté ici se distingue d'autres études qui analysent le voyage féminin comme une occasion d'échapper aux contraintes de genre. 
préface écrite en 1990 de son journal de voyage, de «notre monde à nous, les Français d'Indochine ». Son père, Claude Beaucarnot, originaire du Morvan, était depuis 1920 directeur général de la Société anonyme des Tuileries de l'Indochine, usine située à Hanoi. Sa fille explique : «Connaissant bien l'Indochine, il l'aimait et en estimait ses habitants. Il prit pour épouse, maman, née d'un père breton $[\ldots]$ et d'une mère vietnamienne d'une bonne famille de Ha Dong ». Le couple eut deux enfants, Claudie née en 1924 et Nicole en 1929, et adopta deux jeunes gens métis Paulette et Georges dans cette même décennie ${ }^{3}$. Résidant dans une grande maison près de l'usine de tuileries, la famille évolue au sein d'une société composée d'amis français, de visiteurs étrangers et d'autres familles métisses de l'élite coloniale, comme les Rouelle, Maitam et Leconte ${ }^{4}$. Les deux filles Beaucarnot font leur scolarité au sein du prestigieux Lycée Albert Sarraut où elles étudient l'anglais et le français ; le vietnamien est appris à la maison.

$\mathrm{La}$ famille voyage régulièrement en Indochine et passe fréquemment des vacances à Ninh Binh, une station proche de Hanoi dans les collines. Ces voyages sont facilités par l'amélioration de l'infrastructure touristique (notamment dans les secteurs du transport et du logement), l'Office du tourisme et de la propagande ayant beaucoup œuvré pendant l'entre-deux-guerres pour promouvoir le tourisme comme partie intégrante du développement régional ${ }^{5}$. En 1934, pour des vacances dans une ville d'eau française, la famille

3 Georges Couteau a été adopté par l'intermédiaire de l'Association des orphelins métis, association dont les Beaucarnot étaient membres fondateurs. Del Testa, «The Vacation Diary of Claudie Beaucarnot - Understanding the Journey », p. 5, http://www.bucknell.edu./Beaucarnot/understanding.shtml.

Pour plus d'information sur le statut des enfants métis, voir Saada 2007.

4 La famille Beaucarnot était surtout proche de la famille Rouelle habitant à Nha Trang. M. Rouelle était français et sa femme vietnamienne-indienne. Propriétaire d'une grande plantation de café, Émile Leconte était le père de douze enfants nés de plusieurs femmes vietnamiennes. De son côté, M. Maitaim était un riche industriel vietnamien qui a épousé une femme d'origine franco-vietnamienne.

5 Teston \& Percheron 1932 et Furlough 2002. 
Beaucarnot ne prit pas la route régulière vers Marseille mais fit un détour par le Japon, Hawaii, les États-Unis et le Canada ${ }^{6}$.

Pour leurs vacances d'été en 1943, la famille Beaucarnot quitte Hanoi par le sud au volant de sa voiture, signe de pouvoir colonial dans l'Indochine de ces années-là où la majorité des véhicules assurent un transport collectif. Voyageant le long de la Route Coloniale \#1, elle s'arrête dans des hôtels, chez des amis, comme les Blaick à Phuoc Haï et les Rouelle à Nha Trang, et dans des bungalows touristiques construits à l'initiative du gouvernement et interdits aux Vietnamiens.

Le voyage est l'occasion pour Claudie Beaucarnot de commenter le paysage physique et culturel, puisant dans sa connaissance des régions et de l'ethnographie contemporaine pour interpréter ce qu'elle observe. En voyageant près de Phan Rang, une ville balnéaire au milieu de la côte du sud, elle écrit que c'est «en plein pays Cham. On voit deux tours chames au bord de la route ». Les femmes cham «sont très sales et se mettent sur la tête un genre de turban [...] Ce turban sert de coussinet sur lequel elles posent leurs charges (influence hindoue)» (6 juillet). À Hué, elle mélange l'observation curieuse des tombeaux impériaux avec un récit nourri d'informations historiques afin de créer sa version personnelle du savoir colonial. Après une journée de visites touristiques, la famille profite d'une soirée cosmopolite au cinéma Morin en regardant un film américain. La jeune fille note aussi son appréciation des différentes cuisines locales et régionales, exprimant ainsi son identification et son attachement à tout ce qui est indochinois: "J'adore la cuisine annamite. À mon avis, c'est la meilleure du monde » (6 août).

Le voyage confirme néanmoins son sentiment de différence culturelle, comme lorsqu'elle décrit avec fascination les habitants montagnards près de Dalat, appelés à l'époque les «Moïs» ou les «Sauvages». Leurs corps et leurs caractéristiques physiques sont décrits avec respect et fascination, en suivant une grille contemporaine de différence raciale. Alors que la voiture dépasse un groupe de ces

6 Del Testa 2006: 14-15. Pour le développement touristique dans l'Indochine coloniale, voir Furlough 2006. 
montagnards occupés à des travaux routiers, elle note l'étrangeté de voir ce peuple «fier et indépendant» travaillant pour des étrangers. Dans son journal de vacances, Claudie en parle longuement, usant d'un langage sensuel et racialisé pour décrire vêtements et corps, mais en même temps il y a d'autres personnes qu'elle ne "voit» pas ou qu'elle ne reconnait pas vraiment, comme le "chauffeur", le boy et le «cuisinier» (le bep) qui font partie de sa vie quotidienne. Ceux-ci restent anonymes dans son journal, leur travail et leurs identités décrits par leur seul «nom» descriptif, signe de son identification avec les hiérarchies de classe dans l'empire.

L'identification sociale de Claudie Beaucarnot et son identité personnelle sont étroitement liées à l'Indochine. Tout en vivant et voyageant au sein du monde social et culturel des colons français, elle se revendique à la fois française, vietnamienne et indochinoise. Alors que le voyage implique la volonté d'abandonner des "positions figées $»^{7}$, il donne aussi l'occasion de les affirmer, comme on le voit à ces mots : «Moi, je suis tonkinoise [...] Mais j’aime mon Tonkin plus que tout. J'y suis née. Et bien qu'il me soit agréable de le quitter de temps en temps, je suis si heureuse d'y revenir!» (16 juillet). Le journal de la jeune fille contient d'autres éléments spécifiques qui montrent son attachement à l'Indochine, en particulier la nourriture et le langage. Certes, sa famille se nourrit à la française et observe les pratiques sociales associées à la nourriture, comme le fait de proposer un apéritif aux invités, mais elle mange aussi vietnamien. On voit ici l'influence de la mère qui s'arrête le long du voyage pour acheter du nuôc mam (une sauce fermentée à base d'anchois qui sert de base à la cuisine vietnamienne). L'utilisation du vietnamien dans le journal témoigne de même de sa familiarité avec cette langue 8 .

Pour Claudie Beaucarnot, les vacances d'été ont été un voyage «à l'extérieur » à travers une partie de l'Indochine qui lui était cependant familière, suivi d'un retour «à la maison» au Tonkin. L'écriture du

L’expression «position figée » vient de Said 1991 : 18 (cité dans Thomas 1994 : 6).

8 Le journal montre que la mère de Claudie parlait couramment le vietnamien et que Claudie le connaissait. Je remercie David Del Testa de m'avoir aidée à clarifier cette question. 
journal est l'occasion de montrer son savoir colonial qui distingue les peuples selon les idées contemporaines de race et de statut. Ce voyage à travers l'espace impérial est aussi un exercice de fabrique de soi, lui permettant d'affirmer sa propre identification avec l'Indochine comme lieu de naissance et de vie, ainsi que son identité ni totalement française ni totalement vietnamienne. Certes, le voyage nourrit la curiosité de la jeune fille concernant l'histoire, les peuples et les cultures autres, mais son accès aux lieux de distractions touristiques, au voyage en automobile, aux hôtels français et aux résidences balnéaires renforcent son sentiment d'identification avec les élites coloniales françaises.

Avec la fin de l'Indochine française, Claudie Beaucarnot entreprit un autre voyage en 1949, celui du rapatriement en France. C'est de là, en 1990, qu'elle écrivit la préface de son journal de vacances de 1943, revendiquant l'identité de sa famille comme celle de «vrais Indochinois » et affirmant pour elle et les siens que «ce pays [...] était le nôtre».

\section{Bibliographie}

DEL TESTA David 2002, «The Vacation Diary of Claudie Beaucarnot - Understanding the Journey", Adien Saigon, Au Revoir Hanoi: The 1943 Vacation Diary of Claudie Beaucarnot. Located at : http://www.bucknell.edu/Beaucarnot/intro.shtml

—, 2006, «From concubinage to indochinoise: reading the Beaucarnot diary for new creole identities in French Indochina ». Paper presented at the 2006 meeting of the American Historical Association.

Furlough Ellen, 2006, «Revisiting 'Imperial Eyes': Tourism in French Colonial Indochina ». Paper presented at the Society for French Historical Society, Illinois (available upon request).

-, 2002, «Une leçon des choses: Tourism, Empire, and the Nation in Interwar France, » French Historical Studies, 25, 3, p. 441-473.

SAADA Emmanuelle, 2007, Les enfants de la colonie. Les métis de l'Empire français entre sujétion et citoyenneté, Paris, La Découverte.

SAID Edward, 1991, "Identity, Authority, and Freedom: the Potentate and the Traveler », Transition, 54.

Teston Eugène \& Maurice PerCheron, 1932, L'Indocbine Moderne: Encyclopédie Administrative, Touristique, Artistique et Économique, Paris, Libraire de France.

ThOMAs Nicholas, 1994, Colonialism's Culture: Anthropology, Travel, and Government, Princeton, 1994. 